\title{
Techniques of Facial Synthesis: A Comprehensive Literature Review
}

\author{
Deepti Chandra \\ Shri Shankaracharya College \\ of Engg. \& Technology, Bhilai, \\ Chhattisgarh, India
}

\author{
Sanjeev Karmakar \\ Bhilai Institute of Technology (BIT) \\ Chhattisgarh, Durg 491001, India
}

\author{
Rajendra Hegadi \\ Pragati College of Engg. \& \\ Management \\ Raipur,Chhattisgarh, India
}

\begin{abstract}
As an important area of computer graphics, realistic image synthesis provides principles and techniques for creating realistic imagery through computations based upon models of real-world objects and behaviors. Facial expression is one of the primary communication means of the human. The ultimate goal of realistic image synthesis is to achieve perfect realism -the synthesized images are visually indistinguishable from their real-world counterparts. This area has widespread applications in industry and scientific research including

3D design, omputer animation, scientific visualization and virtual reality Facial expression synthesis has many useful applications in

practice. How to synthesize facial expression images robustly and simply is still a challenging problem. This paper surveys the state of the art in this area.
\end{abstract}

\section{Keywords}

Facial Synthesis, Eigen pictures, FED, Active Appearance Mode, Parameterization.

\section{INTRODUCTION}

As an important area of computer graphics, realistic image synthesis provides principles and techniques for creating realistic imagery through computations based upon models of real-world objects and behaviors. The ultimate goal of realistic image synthesis is to achieve perfect realism -the synthesized images are visually indistinguishable from their real-world counterparts. This area has widespread applications in industry and scientific research including 3D design, computer animation, scientific visualization and virtual reality. In the last twenty-five years, a large number of research papers have been devoted to this area [3]. Nowadays, as people are enjoying the pleasure of realistically duplicating objects and complex scenes in the virtual world, synthesis of the human face has become one of the most promising research fields, because the face appears as the most perceptual knowledge of human beings [2].

Accordingly, facial analysis [4-8] and synthesis [9, 10, 11] have emerged to be two important requirements for a vast array of vision- based applications. Facial analysis refers to the extraction from video sequences of information concerning the location of the head, its pose, and the movement of facial features such as the eyes and the mouth. Facial synthesis refers to the reverse process of animating a facial model using a set of high-level parameters that control the face's gaze, mouth orientation, and pose. Facial analysis would be useful for such applications as eyetracking, facial expression recognition, and visual speech understanding. Facial synthesis would be useful for animating cartoon characters or digital actors. Together, facial analysis and facial synthesis in tandem would be useful for model-based coding applications such as video email and videoteleconferencing, as well as interactive animation of cartoon characters using facial motions [12].

Usually, facial image analysis/synthesis is useful in several applications. Among them is low bandwidth teleconferencing which may involve the real-time extraction of facial control parameters from live video at the transmission site and the reconstruction of a dynamic facsimile of the subject's face at a remote receiver. Teleconferencing and other applications require facial models that are computationally efficient and also realistic enough to synthesize the various nuances of facial structure and motion. Over a decade ago, the anatomy and physics of the human face, especially the arrangement and actions of the primary facial muscles, provide strong constraints and a principled basis for facial image analysis and synthesis [13]. Part of the difficulty of facial image analysis is that the face is highly deformable, particularly around the forehead, eyes, and mouth and these deformations convey a great deal of meaningful information [14]. In facial image analysis/synthesis, two kinds of research directions are usually made in the literature such as, (1) the technique of synthesizing facial pose images, and (1) the technique of synthesizing facial expression images.

In the first technique (facial pose image synthesis), several methods have been proposed in the literature. These methods can be classified into three categories: anatomy based methods, geometry based methods and learning methods. Anatomy based methods build face models by estimating the dynamic facial muscle contractions from a sequence of human face images [3]. It needs a lot of pre-processing, such as registration of corresponding muscle points and setting constraints of muscle contraction to every sample. Although this method is of highly accurate, it is difficult for practical applications. The geometry based method recovers shape from information such as shading, stereo, motion, texture, etc. For example, the shape from shading method (SFS) deals with the recovery from a gradual variation of shading in an image [7]. The SFS method needs to compute the surface orientation map, such as a normal direction or gradient field from image intensity and then reconstruct the surface depth map from the orientation map. Since the SFS is highly dependent on the gradual variation of shading in the image, it is an illposed problem and difficult to find a unique solution without additional constraints. The learning method finds the related objects between pose subspaces by training samples

It is shown that linear transformations can be learned 
exactly from a basis set of 2D prototypical views for linear object classes in [8]. Locally linear Embedding (LLE) is used to learn common hidden structures shared among different pose images to find parameters which control the pose variation [1].

On the other hand, facial expression mapping has been a popular method for generating facial animations. Realistic facial expression synthesis [39] has been one of the most interesting yet difficult problems in computer graphics. In general, the face is capable of generating on the order of 55,000 distinguishable facial expressions with about 30 semantic distinctions. Given an image of a subject's neutral face and another image of this person's face with an expression, the positions of the facial features (eyes, eye brows, mouth, etc.) on both images are located either manually or through some automatic method. The difference vector is then added to the feature positions of a new face to generate the new expression for that face through geometry-controlled image warping [15], [16]. The division of face into the sub regions .For each sub region, image correlation is used to find the best match in the example database and send the index over the network to receiver [17]. Data-driven approach is to segment a face into sub regions thus providing local control to the user [18]. To map one person's expression details to a different person's face. In addition to feature point motions [19], to create 3D animations from a single face image or a video. Their system automatically estimates the $3 \mathrm{D}$ face mesh, pose, and lighting parameters from a single image. It then transfers a 3D facial expression of a different person to the reconstructed face model by mapping the geometric difference vectors [20].

\section{FACIAL EXPRESSION SYNTHESIS}

In this Section, facial expressions synthesis approaches are summarized. Facial modeling and expressions synthesis research falls into two major categories, those based on geometric manipulations and those based on image manipulations [1].

\subsection{Geometric Manipulations}

\subsubsection{Interpolation}

Techniques offer an intuitive approach to facial expressions synthesis. Typically, an interpolation function specifies smooth motion between two key-frames at extreme positions, over a normalized time interval. Although, interpolations are fast and easily generate primitive facial expressions, their ability to create a wide range of realistic facial configurations is severely restricted (Pighin, Hecker, Lischinski, Szeliski \& Salesin, 1998)[1].

\subsubsection{Parameterization}

Techniques for facial expressions (Parke \& Waters, 1996) overcome some of the limitations and restrictions of simple interpolations. Ideally parameterizations specify any possible face and expression by a combination of independent parameter values. Unlike interpolation techniques, parameterizations allow explicit control of specific facial configurations. Combinations of parameters provide a large range of facial expressions with relatively low computational costs. Nevertheless, there is no systematic way to arbitrate between two conflicting parameters, hence parameterization rarely produces natural

human expressions. Another limitation is that the choice of the parameter set depends on the facial mesh topology and, therefore, a complete generic parameterization is not possible. Furthermore, tedious manual tuning is required to set parameter values, and even after that, unrealistic motion or configurations may result.

\subsubsection{Physics-based muscle models}

Fall into three categories: mass spring systems, vector representations, and layered spring meshes.

I. Mass-spring methods propagate muscle forces in an elastic spring mesh that models skin deformation. Forces applied to elastic meshes through muscle arcs generate realistic facial expressions.

II. The vector muscle method models the action of muscles upon skin exploiting a delineated deformation field. The positioning of vector muscles into anatomically correct positions can be a daunting task. No automatic way of placing muscles beneath a generic or person-specific mesh is reported. They are widely used because of their compact representation and independence of the facial mesh structure.

III. Layered spring mesh muscles models detailed anatomical structure and dynamics of the human face (Terzopoulos \& Waters, 1990). This model achieves great realism. However, simulating volumetric deformations with 3D lattices requires extensive computation.

\subsubsection{The Finite Element Method (FEM)}

A numerical approach is for approximating the physics of an arbitrary complex object. An object is decomposed into area or volume elements, each endowed with physical parameters. The dynamic element relationships are computed by integrating the piecewise components over the entire object (Nebel, 2001). They are quite computationally intensive methods.

\subsubsection{Pseudo or simulated muscle}

Modeling produces realistic results by approximating human anatomy, but it is difficult to consider the exact modeling and parameter tuning needed to simulate a specific human facial structure. Muscle forces are simulated in the form of splines, wires, or free form deformations:

\subsubsection{Free form deformation (FFD)}

Deforms volumetric objects by manipulating control points arranged in a 3D lattice. FFDs can deform many types of surface primitives, including polygons; quadric, parametric, and implicit surfaces; and solid models. On the other hand, rational FFD (RFFD) (Karla, Mangili, Thalmann \& Thalmann, 1992) incorporates weight factors for each control point. A Dirichlet free form deformation (DFFD) (Lee, Karla \& Thalmann, 1997) is another free-form variation.

I. Spline pseudo muscle models supports smooth and flexible deformations,allowing localized deformation on the surface, reducing the computational complexity.

II. Wire curves together with a collection of domain curves provide an implicit modeling rimitive (Singh \& Fiume,

1998).

III. Wrinkles: There are two types of wrinkles, 
temporary wrinkles that appear for a short time in expressions, and permanent wrinkles that form over time as permanent facial features. Wrinkles and creases are modeled by physically based modeling with plasticity or viscosity, and texture techniques like bump mapping:

IV. Bump mapping produces perturbations of the surface normals that alter the shading of a surface, either by defining wrinkle functions, or using morphing. Bump mapping was difficult to compute in real time.

V. Physically based wrinkle models use the plastic-visco-elastic properties of the facial skin and permanent skin aging effects (Wu, Thalmann \& Thalmann, 1994). Viscosity is responsible for time dependent deformation, while plasticity is for noninvertible permanent deformation that occurs when an applied force goes beyond a threshold.

VI. Other wrinkles approaches are spline segments and time varying homotopy based on homotomy sweep technique (Moubaraki, Tanaka, Kitamura, Ohya \& Kishimo, 1994).

\subsection{Image Manipulations}

2D and 3D morphing effects a metamorphosis between two target images or models. A 2D image morph consists of a warp between corresponding points in the target images and a simultaneous cross dissolve. Typically, the correspondences are manually selected to suit the needs of the application. The 2D and 3D morphing methods can produce realistic facial expressions, but they share similar limitations with the interpolation approaches.

\subsubsection{Vascular expressions}

Realistic face modeling and animation demand not only face deformation, but also skin color changes that depend on the emotional state of the person. Not much research is reported on this subject. It can be achieved by changing the color of all the polygons during strong emotion (Karla \& Thalmann, 1994), or using texture mapping.

\subsubsection{Model fitting}

Some techniques have been developed, in order to fit a predefined wireframe on a face. This face model can be of any level of detail, but it is usually limited in locating a rough outline of the face and a set of facial movements. Such model-fitting algorithms on faces derived from images are described in (Ahlberg, 2001).

\subsubsection{Expressions using tracking}

The difficulties of other methods in achieving life-like facial expressions led to the performance driven approach where tracked human actors control the expressions. Real time video processing allows interactive animations where the actors observe the animations

they create with their motions and expressions. Accurate tracking of feature points or edges is important to maintain a consistent and life- like quality of expression.

\section{LITRATURE REVIEW OF FACIAL SYNTHESIS MODELS}

Since 1990, there has been a tremendous amount of work on image- based face modeling and animation. In the following,
International Journal of Computer Applications (0975 - 8887)

Volume 61-No.10, January 2013

we review the literature along five different but interconnected threads: statistical models and subspace representation, geometry modeling, appearance modeling , animation, and the modeling of eyes and hair.

\subsection{Statistical models and subspace representation}

In 1987,Sirovichand Kirby [23,24] proposed to use eigenvectors (called eigenpictures) to represent human fac es.In 1991 in their seminar paper[26],Turk and Pentland proposed to use this representation for face recognition, and they call the eigenvectors

"eigenfaces" .T/heir work not only generated much excitement in face recognition but also made Principal Component Analysis an extremely

popular tool in designing statistic models for image analysis and

synthesis.In 1992,Yuille et al.[29] developed a template-based technique for detecting facial features.Their technique can be thought of as extension to snake technique in that local elastic models in snakes are replaced by global deformable templates.In 1995,Cootes et al[30] proposed to use a statistic shape model,called Active Shape $\operatorname{Model}(\mathrm{ASM})$, as a better deformable template. An active shape model is constructed by applying ASM was later extended to the Active Appearance Model(AAM), which consists of a statistic shape model as well as a statistic texture model. In 1997, Vetter and Poggio introduced linear object classes for synthesizing novel view of objects and faces.In 1999, Blanz and Vetter[19] futher extended this idea and introduced a morphable model for 3D face reconstruction from a single image. They used the University of South Florida(USF) dataset of 200

Cyberware scanned faces to construct a statistic shape model and a statistic texture model.Given an input face image,the shape and texture model coefficients can be recovered through an analysis-by-synthesis framework.Since then,both the USF dataset and the notion of the linear object classes have become very popular.

The study of subspace representation of face images under varying illuminations also started to attract much attention in the same period.In 1992,Shashua proposed that, without considering shadows, the images of a Lambertain surface in a fixed pose under varying illuminations live in a three-dimensional linear space.In 1994, Hallinan made a similar observation and proposed to use a low-dimensional represention for image synthesis.In 1995,Murase and Nayar[153] proposed to use low-dimensional appearance manifolds for object recognition under varying illumination conditions.

In 1997,Belhumeur et al.[31] extended Murase and Nayar's appearance manifold method to the case of multiple light sources and shadowing and proposed the illumination cone representation.In

2001,Lee et al.[32] showed that there exists a configuration of nine point light source directions so that the nine image taken under these light source can be used as the basic for the illumination subspace.In the same year,Ramamoorthi and Hanrahan[33] and Basri and Jacobs independently discovered that nine spherical harmonic basis functions

are sufficient to approximate any irradiance map of an object under arbitrary illumination conditions.After that,spherical harmonics became a popular tool for 
illumination modeling[27,28,].

One of the early works on bilinear models was by Brainard and Wandell[24] who tried to isolate the effect of varying illumination from that of varying the surface reflectance.In 19992,D'Zmura[51] proposed to use the bilinear model to recover the surface color(albedo) from image obtained under different illuminations.Around the same period,Tomasi and Kanade[34] published their factorization technique to recover shape and motion from an image sequence.In

19997,Freeman and Tenenbaum proposed techniques to learn bilinear models from training observations and demonstrated how the learned bilinear models can be used for various analysis and synthesis tasks.In

2002,Vasileancu and Terzopoulos[35] proposed a multilinear representation,called TensorFace to model face images with variations

in multiple modes such as identity,expression,illumination, and head

pose.In 2005,they[36] introduced a multilinear independent component analysis framework that generalizes the traditional ICA(Independent Component Analysis) to the multilinear case.

\subsection{Geometry modeling}

In early 1990s, many researchers started to become interested in 3D face reconstruction from images. In 1991,Leclerc and Bobick [37] developed a shape-from-shading technique to reconstruct the 3D face geometry from images where they used stereo processing to initialize the shape-from-shading solver. In 1994,Devernay and Faugeras developed a technique to recover the 3D structure by first computing differential properties from disparity maps, and they applied the technique to face images .In 1996,Proesmans et al.[38] developed a structurelight-based system for acquisition of 3D face geometries. In the same year,Ip and Yin[39] developed a system to construct 3D face model from two orthogonal views.

In 1998,Fau and Miccio [67,68] developed a system to fit an animated face model to noisy stereo data. Given a video sequence,they treat consecutive frame as stereo pair or triplets. They assumed that both the intrinsic and extrinsic camera parameters were known a priori, The obtained strereo data was used as input for 3D model fitting.In 1999,Fua[66] extended the system so that it does not require calibration data.Given a video sequence for which the calibration motion motions are not known,they used a generic face model to regularize the bundle adjustment so that they were able to recover camera motions between successive frames,After that,they generate stereo data fit a face model as in[67].

In 2000 and 2001 , Liu et al.[134,135,193,268] developed a system to construct an animated 3D face model from a video sequence.They used a linear space representation to reduce the number of unknowns in the bundle adjustment process.In 2001, Chen and Medioni developed a stereovision-based facemodeling system using a precalibrated and synchronized camera pair.In 2004,Dimitriejevic et al.[40] developed a structure-frommotion-based system to reconstruct $3 \mathrm{D}$ face models from uncalibrated image sequence

In 2006,Golovinskiy et al.[41] developed an example-based approach to synthesize geometric details.They acquired highresolution face geometries for different ages,genders, and races.The geometries details are extracted and represented with displacement maps.Statistical

models of the displacement maps are obtained and used to synthesize plausible geometric details of a new face.
In 2007, Amberg et al.[42] developed a model-based stereo system to recover the $3 \mathrm{D}$ face geometry as well as the head poses from two or more images taken simultaneously.

\subsection{Appearance modeling}

Another work that started at the end of 1990s was the reflectance and illumination recovery of face images.In 1997,Marschner and Greenberg[43] developed an lighting system for face relighting.Given a camera model,the face geometry and albedo,and a set of basis lights,their system produces a set of basis images for the face under the basis lights.Given a new photograph,their system searches for a linear coefficients are essentially the lighting coefficients.In 1999,Marchner et al.[45] developed an image-based system to measure the Bidirectional Reflectance Distribution Function(BRDF). They assumed that the surface area of an object is curved and all the point on the measured object have the same BRDF.In 2000,Debevec et al.[44] developed a system to capture spatially varying reflectance properties of a human face's skin area.It require 2D array of light sources and a number of synchronized cameras,and the light sources and the cameras need to be calibrated.In 2005, Weyrich et al.[46,47] developed a system to capture and measure a more general bidirectional surface scattering distribution function, which takes into account the translucent component of the skin reflectance.In 2007, Wang et al[48,49] proposed a spatially varying texture morphable model.They divide the image into multiple subrrgions and have a separate texture morphable model for each subregion.The spatial coherence between subregions is modeled as a Markov random field.Their technique is capable of handling harsh lighting conditions that cause cast shadows and saturations, as well as partial occlusions.

\subsection{Animation}

In 1997,Bregler et al.[51] developed a vedio-rewrite system that synthesizes lip-synchronized face animation from speech.This technique was later extended by Brand[50],Ezzat et al.[52], and joshi et al.[53].In 1998,Guenter et al.[54] developed a system with six synchronized cameras to capture 3D facial expressions.In the same year,Pighin et al.[55] used convex combination of the geometries and textures of a person's example facial expressions to generate new facial expressions for the same person.Basically the geometries and textures of a person's expressions. This representation was also used for facial expression tracking[56].In 2001,Liu et al.[57] proposed a technique,called the expression ratio in] mage,to map one person's expression details to a different person's face.Noh and Neuman[58] developed the expression cloning technique to map the geometric motions of one person's expression to a different person.In 2004,Sumner and Popovic[59] developed a technique to transfer detailed geometric deformation between two 3D face meshes.In

2006,Zhang et al.[60] developed a technique to synthesize photorealistic facial expression details from feature point motions.In

2007 ,Song et al developed a general framework for facial expression transfer.Their technique works on both 3D meshes and 2D images. 


\subsection{Eyes and Hair Modeling}

\subsection{1. $\quad$ Eyes}

The amount of research work on modeling face organs such as eyes and mouth has been relatively small.In 1994,Sagar et al.[61] proposed an anatomy-based eye model for surgical simulation.In 2002,Lee et al[62] developed an eye movement model based on eye-tracking data of face-to-face conversations.In 2003, Itti et al.[63] developed a neurobiogical model of visual attention including the eye and head movement.Based on eye gaze tracking data,a visual attention model was built to predict the gaze direction for any given visual stimuli(e.g.,an image).A model for eye and head movement was also developed to generate animations for avatars.

\subsubsection{Hair}

In comparison, hair modeling has recived a lot more attention.One of the earlier works on furry surface rendering was published in 1989 by kajiya

proposed and kay[64].They a volumetric texture

represention,called texel,to render furry surfaces.They generated some impressive rendering result of a teady bear model.In 1992,Anjoyo et al.[65] developed a technique for modeling hair style and dynamic behavior.They derived simple diffential equations that coarsely approximate physics while capturing the aesthetic feature of human hair.In 1995,Stam [66] proposed to use motion vector field for hair modeling by tracing particles through the vector field.The trajectory of the particle

determines the shape of the hair

strand.In 1997,Goldman[67] accelerated Kajiya and Kay's method[68] by using an aggregated lighting model.In 1999, Chen et al.[69] developed a hair rendering system using trigonal prism wisp model.In 2000,Kong and Nakajima[70] proposed a jittering and pseudo shadow technique to improve the realism of hair renderering.

In 2001, $\mathrm{Yu}[71]$ used a 3D vector field for hairstyle modeling.They introduced a set of vector field primitives for both global and local control of hairstyles.In 2002,Chang et al.[72] developed a system to model the interactions of the hair strands by applying dynamics simulation on a sparse set of guide strands.In 2003, Marschner et al. [73] measured lights scattering from hair fibers and proposed a hair shading model that is more accurate than the model proposed by Kay [74].

In 2004,Pais et al[75] developed an image-based technique to capture thither image-based hair geometry by extending the system of[76] to allow multiple view points.In this way,they were able to capture the geometry of the entire hair surface.

In 2005, Wei et al.[77] proposed an image-based approach to capture the hair geometry.In 2006,Bertails et al.[78] proposed to use Kirchhoff's equations for elastic rods to describe the motion of hair strands. The equations are solved representing a hair strand as a piecewise helical rod.In the same year,Moon and Marshner[79] proposed an efficient physically based technique to simulate the multiple scattering of hair based on volumetric photon mapping.

In 2008,Moon et al.[80] improved their previous technique[81] and developed a much faster volumetric rendering method to compute

multiple scattering solutions.

In 2009,McAdams et al.[82] developed a hybrid approach to simulate the dynamics of hair interactions where large-scale interactions are handled with fluid solver while local hair-hair interactions are handled with lagrangian self-collision.Wang et
International Journal of Computer Applications (0975 - 8887)

Volume 61-No.10, January 2013

al.[83] developed an example- based approach for hair geometry synthesis.Given an input

hairstyle,their system can generate a new hairstyle, which has a statistically similar hair strand arrangement and geometric details. Based on their synthesis method,they developed a hair-editing technique that can perform hair detail transfer between hairstyles.

\section{CONCLUSION AND FUTURE DIRECTIONS}

Face image processing has been a fascinating topic for researchers in computer vision,computer graphics,and multimedia.Human face is so interesting to us partly because of its familiarity.The goal of this paper is to provide a systematic treatment of the technologies that have been developed in this field.In this paper, after introducing what is Facial expression synthesis and its applications in different domains, we present several facial modeling approaches.. we describe and survey the issues associated with facial expressions analysis and synthesis. Analysis of human facial expressions consists of three steps: face detection (tracking), facial feature extraction and facial classification. Generation of facial expressions can be summarized as follows. First, an individual specific model is obtained and fitted into the prearranged prototype mesh. Second, the constructed individual facial model is deformed to produce facial expression. Wrinkles and vascular effects are also considered for added realism. The goal of research related to the synthesis, achieving realism in real time in automated way, has not been reached yet. In the future, we plan to develop a face expression model that synthesis the face expression. Initially, various expressions of different person are taken and we are preprocessing the images and applying the landmark points to find the deviations using two procedures to train the neural network. The neural network is then used to create the desired face reaction of the neutral face which we are giving as input. For the experimentation, we have used the standard database and the performance is analyzed with the various type of expressions.

\section{REFERENCES}

[1] Stelios Krinidis, Ioan Buciu and Ioannis Pitas" Facial expression analysis and synthesis: A survey" Inter.Conf on Human Computer Interaction HCI 2003.

[2] Yun Sheng, Abdul H. Sadka, Ahmet M. Kondoz, "Automatic Single View-Based 3-D Face Synthesis for Unsupervised Multimedia Applications", IEEE Transactions On Circuits And Systems For Video Technology, Vol. 18, No. 7, July 2008.

[3] Yinlong Sun, "A Spectrum-Based Framework for Realistic Image Synthesis", Thesis, Simon Fraser University, 2000.

[4] Li-an Tang, and Thomas S.Huang, "Automatic Construction of 3D Human Face Models Based on 2D Images", Proceedings of ICIP'96, III, pp. 467-470, 1996.

[5] Y.C.Lee, D.Terzopoulos and Keith Waters. "Realistic modeling for facial animation", In SIGGRAGH'95 Conference Proceedings, pp.55-62, Los Angeles, 1999.

[6] P. Ekman and W.V. Friesen, "Manual for the Facial Action Coding System", Palo Alto: Consulting Psychologists, 1977. 
[7] Arman Savran, Neşe Alyüz, Hamdi Dibeklioğlu, Oya Çeliktutan, Berk Gökberk, Bülent Sankur, Lale Akarun, "Bosphorus Database for 3D Face Analysis", Biometrics and Identity Management, Springer-Verlag Berlin, 2008.

[8] P. Ekman, "Unmasking the Hnman Face", New York: Prentice-Hall, 1971.

[9] Santanu Halder, Debotosh Bhattacharjee, Mita Nasipuri, "Face Synthesis (FASY) System for Generation of a Face Image from Human Description", IEEE Region 10 Colloquium and the Third International Conference on Industrial and Information Systems, Kharagpur, 2008.

[10] Volker Blanz, Thomas Vetter, "A Morphable Model for the Synthesis of 3D Faces", Proceedings of the 26th annual conference on Computer graphics and interactive techniques, 1999.

[11] Salvador E. Ayala-Raggi, Leopoldo Altamirano-Robles and Janeth Cruz-Enriquez, "Face Image Synthesis and Interpretation Using 3D Illumination-Based AAM Models", New Approaches to Characterization and Recognition of Faces, InTech, 2011.

[12] Volker Blanz, Thomas Vetter, "A Morphable Model for the Synthesis of 3D Faces", Proceedings of the 26th annual conference on Computer graphics and interactive techniques, 1999.

[13] Salvador E. Ayala-Raggi, Leopoldo Altamirano-Robles and Janeth Cruz-Enriquez, "Face Image Synthesis and Interpretation Using 3D Illumination-Based AAM Models", New Approaches to Characterization and Recognition of Faces, InTech, 2011.

[14] Tony Ezzat, Tomaso Poggio, "Facial Analysis and Synthesis Using Image-Based Models", Proceedings of the Workshop on the Algorithmic Foundations of Robotics, August 1996.

[15] D. Terzopoulos and K. Waters. "Analysis and synthesis of facial image sequences using physical and anatomical models", IEEE Transactions on Pattern Analysis and Machine Intelligence, Vol: 15, No: 6, pp: 569-579, 1993.

[16] Demetri Terzopoulos, Yuencheng Lee and M. Alex O. Vasilescu, "Model-Based and Image-Based Methods for Facial Image Synthesis, Analysis and Recognition", Proceedings of the 6th IEEE International Conference on Automatic Face and Gesture Recognition (FG'04), Seoul, Korea, May, 3-8, 2004.

[17] T. Beier and S. Neely, "Feature-Based Image Metamorphosis," Computer Graphics, pp. 35-42, July 1992.

[18] [18] P. Litwinowicz and L. Williams, "Animating Images with Drawings," Computer Graphics, pp. 235-242, Aug. 1990.

[19] S. Toelg and T. Poggio, "Towards an Example-Based Image Compression Architecture for Video-Conferencing," TechnicalReport 1494, MIT, 1994.

[20] P. Joshi, W.C. Tien, M. Desbrun, and F. Pighin, "Learning Controls for Blend Shape Based Realistic Facial Animation," Proc. Symp. Computer Animation (SCA '03), pp. 187-192, July 2003.

[21] Z. Liu, Y. Shan, and Z. Zhang, "Expressive Expression Mapping with Ratio Images," Computer Graphics, Proc. Ann. Conf. Series, pp. 271-276, Aug. 2001.

[22] [V. Blanz, C. Basso, T. Poggio, and T. Vetter, "Reanimating Faces in Images and Video," Proc. Eurographics Conf., 2003

[23] M Kirby and M.Sirovich.Application of the Karhunen-Loeve procedure for the characterization of human faces.IEEE
International Journal of Computer Applications (0975 - 8887)

Volume 61-No.10, January 2013

Transactions on Pattern Analysis and machine intelligence 1990.

[24] M Sirovich and M Kirby.Low-dimensional procedure for the characterization of human faces.Jurnal of the Optical Society of America pp519-524,1987.

[25] R.Ramanmoorthi.and P.Hanrahan.An efficient representation for irradiance environment maps.In SIGGRAPH,pp 497-500,2001.

[26] M Turk and A.Pentland.Eigenfaces for recongnition.Jurnal of Congnitive Neuroscience pp7196,1991

[27] YWang,1.Zhang,Z,liu,G.Hua,Z.Wen,Z.Zhang,and samaras .Face re-lighting from a single image under harsh lighting conditions.in IEEE Conference on Computer Vision and Partern Recgnition,Minnesota,June 2007.

[28] Y.Wang,L.Zhang,Z.Liu,G.Hua,Z.Wen,Z.Zhang,and D Samaras.Face re-lighting from a single image under arbitrary unkown lighting conditions.IEEE Transaction on Pattern Recognition and Machine Intelligence,319110;1968-1984,2009

[29] Y.Wang,L.Zhang,Z.Liu,G.Hua,Z.Wen,Z.Zhang,andD Samaras.Face re-lighting from a single image under arbitrary unkown lighting conditions.IEEE Transaction on Pattern Recognition and Machine Intelligence,319110;1968-1984,2009

[30] A. L. Yuille, D. S. Cohen, and P. W. Hallinan. Feature extraction from faces using deformable templates. International Journal of Computer Vision, 8(2):99-112, 1992.

[31] T. F. Cootes, C. J. Taylor, D. H. Cooper, and J. Graham. Active shape models - Their training and application. Computer Vision and Image Understanding, 61(1):18-23, 1995.

[32] P. Belhumeur, J. Hespanha, andD. Kriegman. Eigenfaces vs. fisherfaces: Recognition using class specific linear projection. IEEE Transactions on Pattern Analysis and Machine Intelligence, 19(7):711$720,1997$.

[33] K.-C. Lee, J. Ho, and D. Kriegman. Nine points of light: Accquiring subspaces for face recognition under variable lighting. In IEEE Conference on Computer Vision and Pattern Recognition, 357-362, Kauai, HI, USA, 2001.

[34] R. Ramamoorthi and R Hanrahan. An efficient representation for irradiance environment maps. In SIGGRAPH, pages 497-500, 2001.

[35] C. Tomasi and T. Kanade. Shape and motion from image streams under orthography:A factorization method. International Journal of Computer Vision, 9(2):137- 154,1992.

[36] M. A. O. Vasilescu and D. Terzopoulos. Multilinear analysis of image ensembles: Tensorfaces. In European Conference on Computer Vision, Copenhagen, Denmark, May 2002.

[37] M. A. O. Vasilescu and D. Terzopoulos. Multilinear independent component analysis. In IEEE Conference on Computer Vision and Pattern Recognition, San Diego, CA, USA, 2005 
[38] Y. G. Leclerc and A. F. Bobick. The direct computation of height from shading. In IEEE Conference on Computer Vision and Pattern Recognition, Maui, Hawaii, June 1991.

[39] M. Proesmans, L. V. Cool, and A. Oosterlinck. Active acquisition of $3 \mathrm{~d}$ shape for moving objects. In International Conference on Image Processing, Lausanne, Switzerland, Sept. 1996.

[40] H. H. S. Ip and L. Yin. Constructing a 3d individualized head model from two orthogonal views. The Visual Computer, (12):254-266, 1996.

[41] M. Dimitrijevic, S. Ilic, and P. Fua. Accurate face models from uncalibrated and ill-lit video sequences. In IEEE Conference on Computer Vision and Pattern Recognition, volume II, pages 1034-1041, 2004.

[42] A. Golovinskiy, W. Matusik, H. Pfister, S. Rusinkiewica, and T. Funkhouser. A statistical model for synthesis of detailed facial geometry. ACM Transaction on Graphics, 25(3): 1025$1034,2006$.

[43] B. Amberg, A. Blake, A. Fitzgibbon, S. Romdhani, and T. Vetter. Reconstructing high quality face-surfaces using model based stereo. In International Conference on Computer Vision, Rio de Janeiro, Brazil, October 2007.

[44] S. R. Marschner and D. P. Greenberg. Inverse lighting for photography. In IST/SID Fifth Color Imaging Conference, Scattsdale, Arizona, USA, 1997.

[45] S. R. Marschner, B. Guenter, and S. Raghupathy. Modeling and rendering for realistic facial animation. In Rendering Techniques, pages 231-242. Springer, 2000.

[46] S. R. Marschner, S. Westin, E. Lafortune, K. Torance, and D. Greenberg. Image-based brdf measurement including human skin. In Rendering Techniques, pages 139- 152， Granada, Spin, 1999.

[47] T. Weyrich, W. Matusik, H. Pfister, B. Bickel, C. Donner, C. Tu, J. McAndless, J. Lee, A. Ngan, H. W. Jensen, and M. Gross. Analysis of human faces using a measurement-based skin reflectance model. ACM Transaction on Graphics, 25(3): $\quad$ 1013-1024, 2006.

[48] T. Weyrich, W. Matusik, H. Pfister, J. Lee, A. Ngan, H. W. Jensen, and M. Gross. A measurement-based skin reflectance model for face rendering and editing. In Technical Report, TR2005-046, Mitsubishi Electric Research Laboratories, Cambridge, MA, USA, 2005.

[49] Y. Wang, Z. Liu, G. Hua, Z. Wen, Z. Zhang, and D. Samaras. Face re-lighting from a single image under harsh lighting conditions. In IEEE Conference on Computer Vision and Pattern Recognition, Minneapolis, Minnesota, June 2007.

[50] Y. Wang, L. Zhang, Z. Liu, G. Hua, Z. Wen, Z. Zhang, and D. Samaras. Face re lighting from a single image under arbitrary unknown lighting conditions. IEEE Transaction on Pattern Recogniton and Machine Intelligence, 31(11): 19681984, 2009

[51] M. Brand. Voice puppetry. In SIGGRAPH, pages 2128,1999 .

[52] C. Bregler, M. Covell, and M. Slaney. Video rewrite: Driving visual speech with audio. In SIGGRAPH, pages 353-360, 1997.

[53] T. Ezzat, G. Geiger, and T. Poggio. Trainable videorealistic speech animation. In SIGGRAPH'O2, pages 388-398, 2002.

[54] P. Joshi, W. C. Tien, M. Desbrun, and F. Pighin. Learning controls for blend shape based realistic facial animation. In
International Journal of Computer Applications (0975 - 8887)

Volume 61-No.10, January 2013

Proceedings of the Symposium on Computer Animation (SCA'03), pages 187-192, San Diego, CA, USA, July 2003.

[55] B. Guenter, C. Grimm, D. Wood, H. Malvar, and F. Pighin. Making faces. In SIGGRAPH, pages 55-66, 1998.

[56] F. Pighin, J. Hecker, D. Lischinski, R. Szeliski,.and D. H. Salesin. Synthesizing realistic facial expressions from photographs. In SIGGRAPH, pages 75-84,1998.

[57] F. Pighin, D. H. Salesin, and R. Szeliski. Resynthesizing facial animation through $3 \mathrm{~d}$ modelbased tracking. International Conference on Computer Vision, pages 143-150, Kerkyra, Corfu, Greece, 1999

[58] [57] Z. Liu, Y. Shan, and Z. Zhang. Expressive expression mapping with ratio images. SIGGRAPH, pages 271-276, 2001.

[59] [58] J. Noh and U. Neumann. Expression cloning. In SIGGRAPH, pages 277-288, 2001

[60] R. W. Sumner and J. Popovic. Deformation tranfer for triangle meshes. In SIGGRAPH, pages 399-405, 2004.

[61] Q. Zhang, Z. Liu, B. Guo, D. Terzopoulos, and H.-Y. Shum. Geometry-driven photorealistic facial expression synthesis. IEEE Transaction on Visualization and Computer Graphics, 12(1):48-60, 2006.

[62] M. A. Sagar, D. Bullivant, G. D. Mallinson, P. J. Hunter, and I. W. Hunter. A virtual environment and model of the eye for surgical simulation. In SIGGRAPH, pages 205- 212,1994.

[63] [62] S. P. Lee, J. B. Badler, and N. I. Badler. Eyes alive. In SIGGRAPH, pages 637- 644, 2002.

[64] L. Itti, N. Dhavale, and F. Pighin. Realistic avatar eye and head animation using a neurobiological model of visual attention. In Proceedings of SPIE, pages 64-78, San Diego, CA, USA, August 2003.

[65] J.JL Kajiya and T. L. Kay. Rendering fur with three dimensional textures. In SIGGRAPH, pages 271-280, July 1989.

[66] K. Anjyo, Y. Usami, and T. Kurihara. A simple method for extracting the natural beauty of hair. In SIGGRAPH, pages 111-120,1992.

[67] J. Stam. Multi-scale stochastic modeling of complext natural phenomena. Ph.D. thesis, University of Toronto, 1995.

[68] D. B. Goldman. Fake fur rendering. In SIGGRAPH, pages 127-134, 1997

[69] J.JL Kajiya and T. L. Kay. Rendering fur with three dimensional textures. In SIGGRAPH, pages 271-280, July 1989.

[70] L. Chen, S. Saeyor, H. Dohi, and M. Ishzuka. A system ofpd hairstyle synthesis based on the wisp model The Visual Computer, 15(4): 159-170, 1999.

[71] W. Kong and M. Nakajima. Hair rendering by jittering and pseudo shadow. In Computer Graphics International, Geneva, Switzerland, June 2000.

[72] Y. Yu. Modeling realistic virtual hairstyles. In Pacific Graphics, pages 295-304,October 2001.

[73] J. T. Chang, J. Jin, and Y. Yu. A practical model for hair mutual interactions. In Symposium on Computer Animation, pages 73-80, 2002 
[74] S. Marschner, H. W. Jensen, M. Cammarano, S. Worley, and P. Hanrahan. Light scattering from human hair fibers. ACM Transaction on Graphics, 22(3):780-791, 2003.

[75] J.JL Kajiya and T. L. Kay. Rendering fur with three dimensional textures. In SIGGRAPH, pages 271-280, July 1989.

[76] S. Paris, H. M. Briceno, and R X. Sillion. Capture of hair geometry from multiple ;images. ACM Transaction on Graphics, 23(3):712-719, August 2004.

[77] S. Grabli, F. Sillion, S. R. Marschner, and J. E. Lengyl. Image-based hair capture by inverse lighting. In Graphics Interface, pages 51-58, Calgary, Alberta, May 2002.

[78] Y. Wei, E. Ofek, L. Quan, and H.-Y. Shum. Modeling hair from multiple views. ACM Transaction on Graphics, 24(3):816-820, 2005.

[79] F. Bertails, B. Audoly, M.-P. Cani, B. Querleux, F. Leroy, and J.-L. Leveque. Super-helices for predicting the dynamics of natural hair. ACM Transaction on Graphics, 25(3): 11801187, 2006.
[80] J. T. Moon and S. R. Marschner. Simulating multiple scattering in hair using a photon mapping approach. ACM Transaction on Graphics, 25(3): 1067-1074, 2006.

[81] J. T. Moon, B. Walter, and S. R. Marschner. Efficient multiple scattering in hair using spherical harmonics. ACM Transaction on Graphics, 27(3):31:1-31-7, 2008.

[82] J. T. Moon and S. R. Marschner. Simulating multiple scattering in hair using a photon mapping approach. ACM Transaction on Graphics, 25(3): 1067-1074, 2006.

[83] A. McAdams, A. Selle, K. Ward, E. Sifakis, and J. Teran. Detail preserving contin uum simulation of stright hair. ACM Transaction on Graphics, 28(3):62:1-62:6, 2009

[84] L. Wang, Y. Yu, K. Zhou, and B. Guo. Example-based hair geometry synthesis. ACM Transaction on Graphics, 28(3):56:1-56:9, 2009. 